\title{
Light Optical Microscopy Based Automated 3D Serial Sectioning Enables Defect Analysis in Large Volumes of Additively Manufactured Alloy Samples
}

\author{
Veeraraghavan Sundar ${ }^{1}$, Bryan Turner ${ }^{1}$, Satya Ganti $^{1}$ and William Davis ${ }^{1}$ \\ 1. UES Inc., Robo-Met.3D Services, Dayton, OH.
}

Additive manufacturing (AM) processes allow components to be directly produced from computer aided design (CAD) models by dividing them into thin two dimensional (2D) slices, which are built sequentially on top of one another [1]. Porosity plays a critical role in determining the mechanical behavior of additively and conventionally manufactured metal components. Defects such as voids decrease the strength and fatigue life of these components, which can limit the application of AM. Until the advent of serial sectioning, classical stereological methods that extrapolate information from 2D images were used to quantify porosity from microstructural analyses. Serial sectioning is a practical and direct method of obtaining 3D microstructures, especially when automated [2].

In this study, we used the Robo-Met.3D ${ }^{\circledR}$ system to investigate the quantification of porosity in additively manufactured and conventional alloy components. Robo-Met.3D is a fully automated serial sectioning system that generates $2 \mathrm{D}$ optical microstructural data for $3 \mathrm{D}$ reconstruction in solid materials. Recent studies suggest some advantages of light optical microscopy based methods over nondestructive methods such as laser ultrasound and CT evaluations for additively manufactured samples [3][4].

Two samples of $\mathrm{Ti} 6 \mathrm{Al} 2 \mathrm{Sn} 4 \mathrm{Zr} 2 \mathrm{Mo}$ and $\mathrm{Ti} 6 \mathrm{Al} 4 \mathrm{~V}$ manufactured by powder bed fusion (PBF) and a conventionally processed Inconel 100 sample, were analyzed for this study. Samples were excised from larger component builds, and were conventionally mounted in metallographic mounts $(\sim 38 \mathrm{~mm}$ diameter x 25mm height) for automated serial sectioning. The IN 100 sample was etched with Kalling's reagent to enhance contrast, while the others were examined as polished.

Optical images were automatically acquired with the microscope built into the Robo-Met.3D system. Acquisition parameters are shown in Table 1. Binary images for $2 \mathrm{D}$ analysis were made by selecting pixel intensity threshold using Fiji/ImageJ, and were stacked and aligned using Fiji. Next, 3D datasets including microstructure and porosity defects were reconstructed and visualized in 3D using Image-Pro Premier 3D software, version 9.3. Two of the resultant 3D renderings are shown in Figure 1, visualizing size-classified defect distributions and microstructure when etched.

The pore size distributions from the complete 3D experimental datasets captured with Robo-Met.3D are calculated and summarized in Table 3. We compared Robo-Met.3D direct measurements of the pore size distribution in a volume of material in 2D based on classical stereology and in 3D based on direct observation of the volume. For selected sections in the volume analyzed, equivalent spherical diameter (ESD) was calculated, and compared to the volumetric porosity average calculated from the entire 3D volume. The variation in the porosity measure calculated from each $2 \mathrm{D}$ slice in this selection was significant. For the Ti6242 sample, the minima and maxima of estimates based on analysis of a single slice varied from $-19 \%$ to $+17 \%$ below and above the mean of the $3 \mathrm{D}$ estimates, respectively. Using a similar approach, ranges of -53 to $+133 \%$ and $-23 \%$ to $+35 \%$ were calculated for the Ti64 and the IN100 samples, respectively. 
In this analysis, three materials were successfully serial sectioned over large, representative cubic millimeter volumes, for porosity analysis. Single 2D slices were analyzed for porosity, and were found to have high variability in porosity estimate results depending on the section analyzed. The visualization using serial sectioning methods such as Robo-Met.3D provided accurate representations of microstructure and porosity, which are imperative to correctly detect pores of critical size for fatigue life prediction, especially for additively manufactured parts.

\section{References:}

[1] Sames, W. J., et al, International Materials Reviews 61(5) (2016), p. 315.

[2] DeHoff, R., Journal Of Microscopy 131(3) (1983), p. 259.

[3] Everton, S. et al, TMS 2017 146th Annual Meeting \& Exhibition Supplemental Proceedings (Springer) p. 105.

[4] M A Groeber et al, IOP Conf. Ser.: Mater. Sci. Eng. 219 (2017), p. 012002.

\begin{tabular}{|c|c|c|c|c|c|}
\hline & $\begin{array}{c}\text { Resolution } \\
\text { Overall } \\
\text { Material }\end{array}$ & $\begin{array}{c}\text { in } \mathbf{x}-\mathbf{y} \\
\text { Magnification }\end{array}$ & $\begin{array}{c}\text { Nectioning Rate } \\
\text { (microns) }\end{array}$ & $\begin{array}{c}\text { Sections } \\
\text { Analyzed }\end{array}$ & $\begin{array}{c}\text { Dimensions } \\
\text { Analyzed (microns) }\end{array}$ \\
\hline Ti6242 & $100 \mathrm{X}$ & 1.08 & 5.5 & 70 & $3000 \times 4500 \times 385$ \\
\hline Ti64 & $100 \mathrm{X}$ & 1.08 & 2.3 & 150 & $3439 \times 1590 \times 345$ \\
\hline IN 100 & $100 \mathrm{X}$ & 1.08 & 2.0 & 120 & $1000 \times 1000 \times 240$ \\
\hline
\end{tabular}

Table 1. Serial Sectioning Data Acquisition Parameters
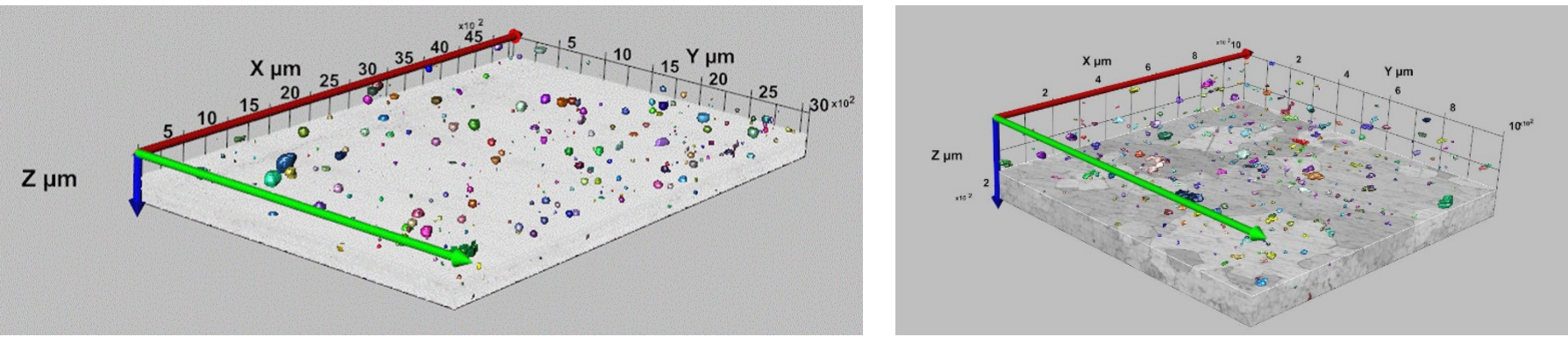

Figure 1. 3D Microstructure and Porosity Distribution for Ti6242 (left) and etched IN 100 (right)

\begin{tabular}{|c|c|c|c|c|c|c|}
\hline Material & Minimum & Maximum & Mean & $\begin{array}{c}\text { 25th } \\
\text { Percentile }\end{array}$ & Median & $\begin{array}{c}\text { 75th } \\
\text { Percentile }\end{array}$ \\
\hline Ti6242 & 5.5 & 149.7 & 40.1 & 17.8 & 31.4 & 58.9 \\
\hline Ti64 & 8.69 & 47.0 & 16.4 & 11.6 & 13.9 & 18.5 \\
\hline IN 100 & 5.1 & 37.1 & 10.3 & 7.05 & 8.5 & 11.7 \\
\hline
\end{tabular}

Table 3. 3D Porosity Statistics for Samples, Microns 